\title{
MECHANICAL PROPERTIES OF THE CELLULAR STRUCTURES BASED ON THE ISOTRUSS UNIT CELL ${ }^{\circledR}$
}

\author{
SEYEDKAMAL JALALI ${ }^{1}$, AMIR YEKTA ${ }^{2}$, AND NICOLA M. PUGNO ${ }^{1,3}$ \\ ${ }^{1}$ Laboratory of Bio-Inspired, Bionic, Nano, Meta Materials \& Mechanics, \\ Department of Civil, Environmental and Mechanical Engineering, Università di Trento, via Mesiano, \\ 77, I-38123 Trento, Italy, seyedkamal.jalali@unitn.it \\ ${ }^{2}$ Department of Automotive Engineering, Iran University of Science and Technology, Tehran, Iran, \\ yekta_a@auto.iust.ac.ir \\ ${ }^{3}$ Griffith Theory Centenary Lab, School of Engineering and Materials Science, Queen Mary \\ University of London, Mile End Road, London E1 4NS, UK, Nicola.pugno@unitn.it
}

Keywords: Cellular Structure, Isotruss, FEM, Biomimetic Structure, REV, Unit Cell.

Summary. The present work aims to investigate the mechanical properties of the cellular structure based on the Isotruss unit cell by implementing the finite element method (FEM) introducing a representative elementary volume (REV). As an orthotropic cellular structure, the three principal Young's modulus along the longitudinal and transversal directions are evaluated. The influence of the geometrical parameters on the elastic properties is studied in detail. Results reveal that Young's modulus in two transverse directions are almost close, showing a $12 \%$ difference. Besides, the proposed unit cell presents the ability to tune the elasticity along with the main directions. It is seen that increasing the angle of helical pitch, increases the longitudinal Young's modulus while decreases the transverse ones that suggest a considerable possibility for optimal designs. As an example, mimicking Young's modulus of the human cortical bone as an orthotropic material is explored and it is seen that the presented cellular structure based on the Isotruss unit cell can successfully fit.

\section{INTRODUCTION}

In the last decade, additive manufacturing (AM) techniques have developed to a commercial scale. AM offers fabrication of complex geometries for tailoring various types of properties simultaneously. The form-freedom makes it the overriding candidate for the fabrication of functional parts, especially in cutting-edge technologies. The Isotruss grid structure which supports loads in a highly efficient manner was first introduced for aerospace applications. It has a porous tubular geometry whose longitudinal members are parallel to the central axis while helical members spiral around the tubular structure [1]. The one-dimensional repetition of the Isotruss unit cell along its central axis is extensively reported as a long tubular grid structure. The reason for such repetition is that this geometry is ideal for manufacturing by the filament winding process to make a composite structure. However, its application as the unit cell of a porous cellular structure has not been addressed yet. Its tubular nature may make it a candidate 
as the unit cell of porous bone implants thanks to its similarity to the osteon tubular nature.

The present work investigates the mechanical properties of the cellular structure based on the Isotruss unit cell by implementing the finite element method (FEM) introducing a representative elementary volume (REV). As an orthotropic cellular structure, the three principal Young's modulus along the longitudinal and transversal directions are evaluated and the effect of geometrical parameters is studied in detail [2].

\section{MATERIALS AND METHODOLOGY}

\subsection{Three-Dimensional modeling}

Figure 1 introduces the proposed unit cell based on six-node Isotruss geometry as a candidate for cellular solids. In planar section 1-2, the unit cell is surrounded by a hexagon of length $a$ while its height along direction 3 , named bay size, is $b$. six axial struts are laid along direction 3 and twelve helical struts intersect them. The diameter of all struts is considered as $d$. In Figure 2 the corresponding cellular solid formed by repeating this unit cell is shown.
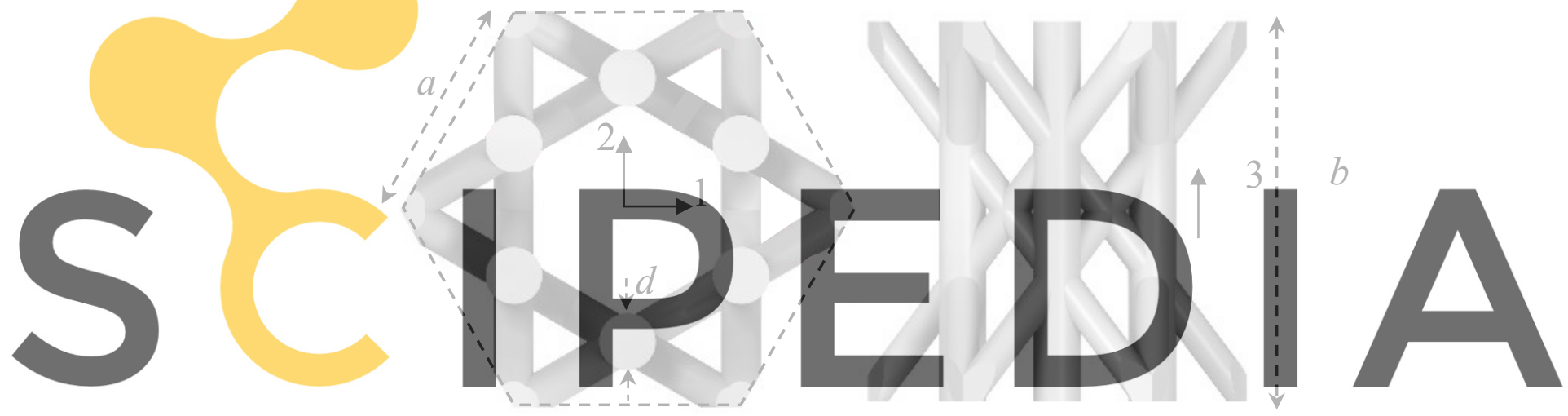

Side and top view of the unit cell of cellular solid based on Isotruss geometry

Register for free at https//www.scipedia.com to download the version without the watermark
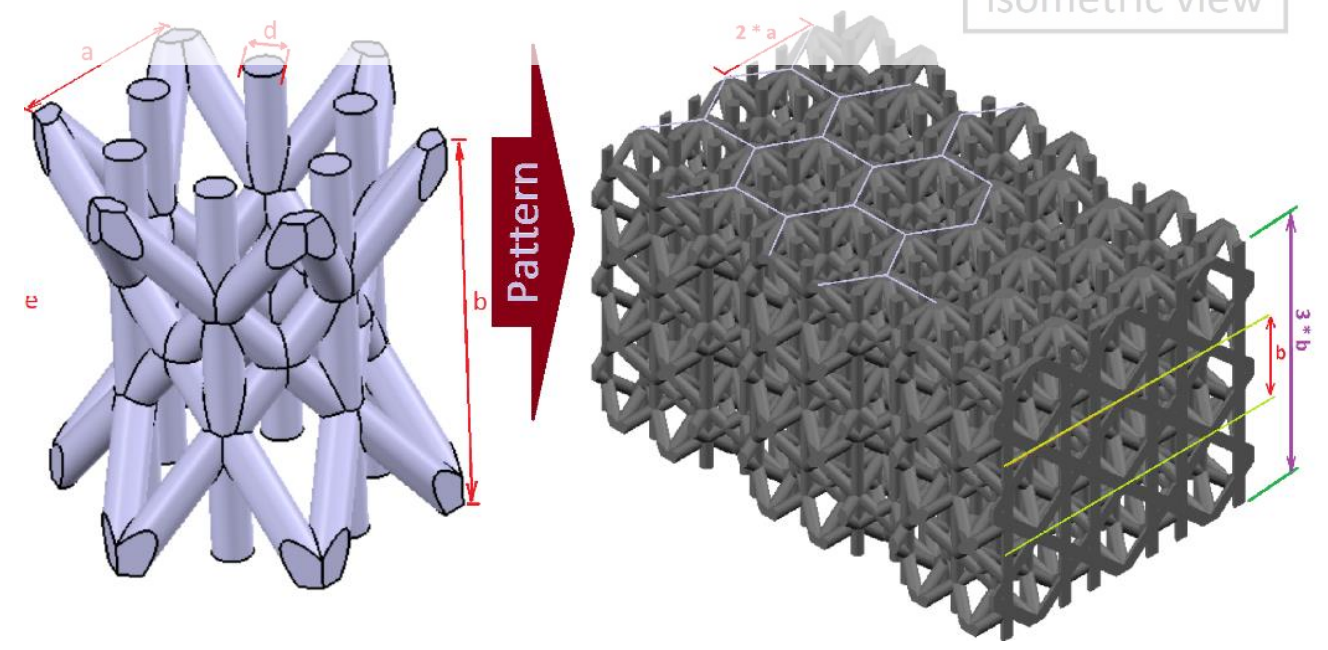

Figure 1: Cellular solid formed by extending the Isotruss unit cell in three-dimensions.

To determine principal Young's modules $\left(E_{1}, E_{2}\right.$, and $E_{3}$ Which represent Young's modulus 
in directions of 1,2 , and 3 , respectively) of this porous lattice cellular structure, models with different geometrical were created in CATIA software. To perform the FEM simulations, a rectangular cube-shaped Representative Elementary Volume $(R E V)$ is defined which is shown in Fig. 2. Rectangular cube-shaped volumes, unlike the hexagonal prismatic geometries, are ideal for applying force and measuring displacements in further steps.

\subsection{FEM analysis}

Considering the defined REV, three principal Young's modules are evaluated via FEM. Boundary conditions are defined by fixing appropriate sides to rigid planes in any analysis and applying load and B.C.s to these rigid planes as shown in Table 1. It is noted that ABAQUS's standard (implicit) is selected as the solver. The corrosion-resistant Ti6A14V alloy with Young's modulus $E_{\text {bulk }}=114 \mathrm{GPa}$ and Poisson's ratio $v_{\text {bulk }}=0.3$ is considered as the bulk material in this analysis [3]. For meshing the specimens, the C3D10 element type, 10-node quadratic tetrahedron, is selected (see Fig. 3). For the possibility of comparison, a conventional isotropic cubic cellular structure (see Fig. 4), which is widely used due to its reasonable properties as well as ease of $3 \mathrm{D}$ modeling and manufacturing is analyzed to evaluate the potential advantages of the presented unit cell based on Isotruss geometry.
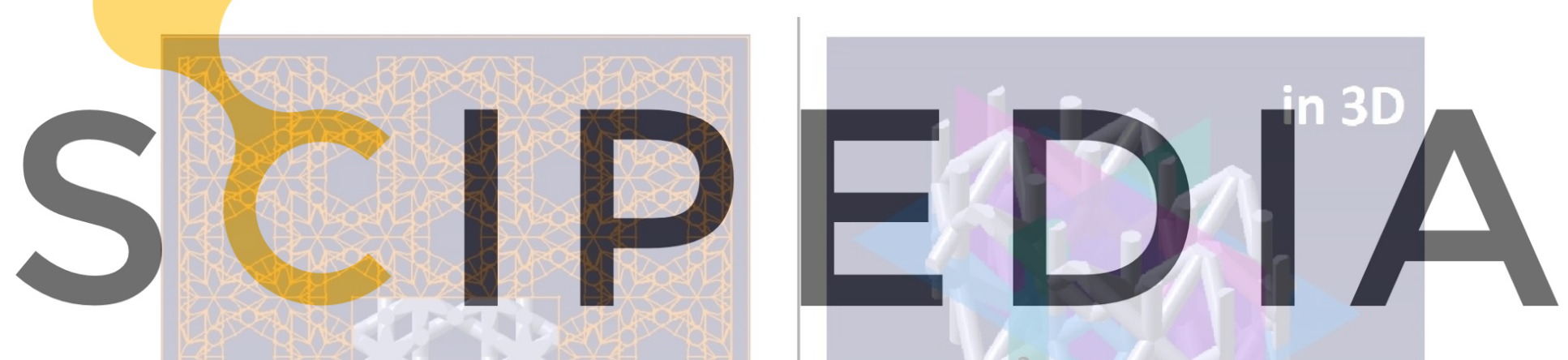

Register for free at https//www.scipedia.com
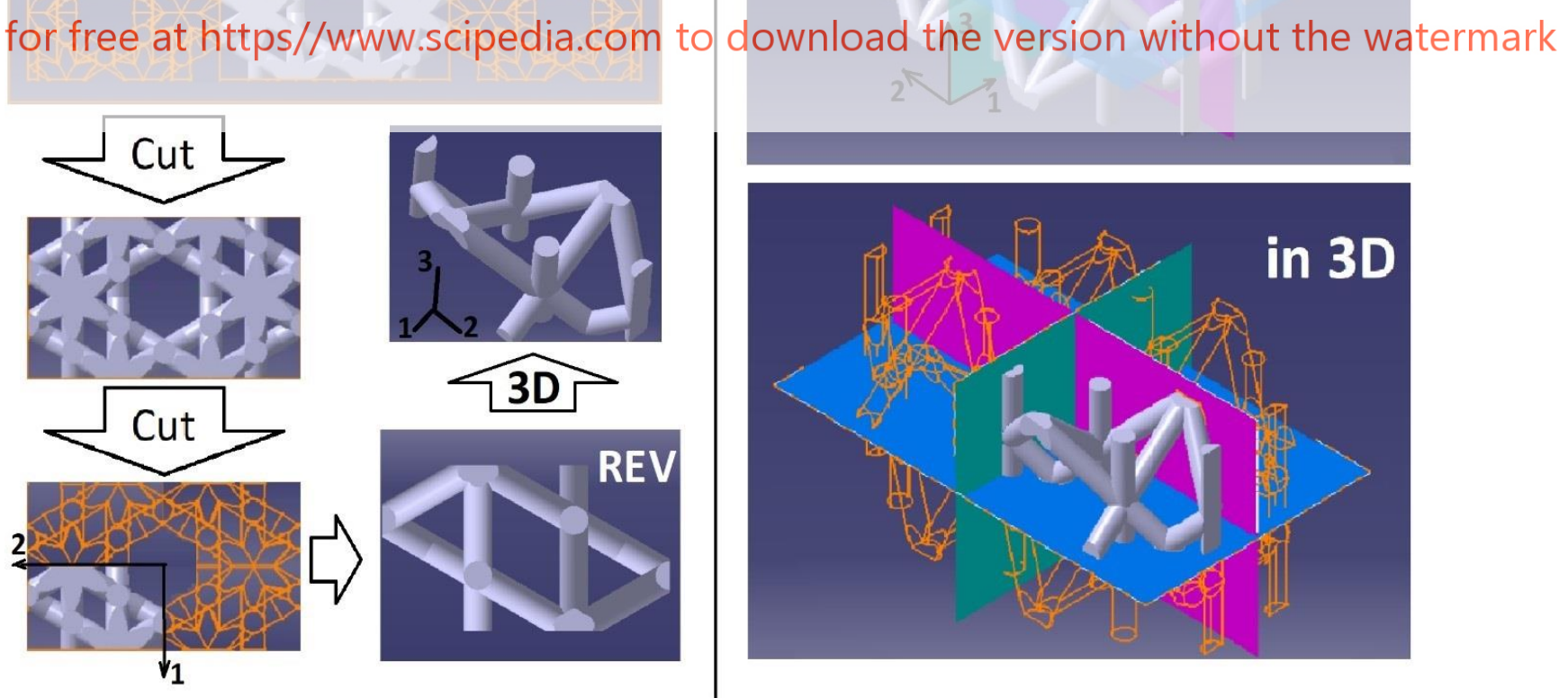

Figure 2: Determination of a rectangular cube shaped representative elementary volume (REV). 


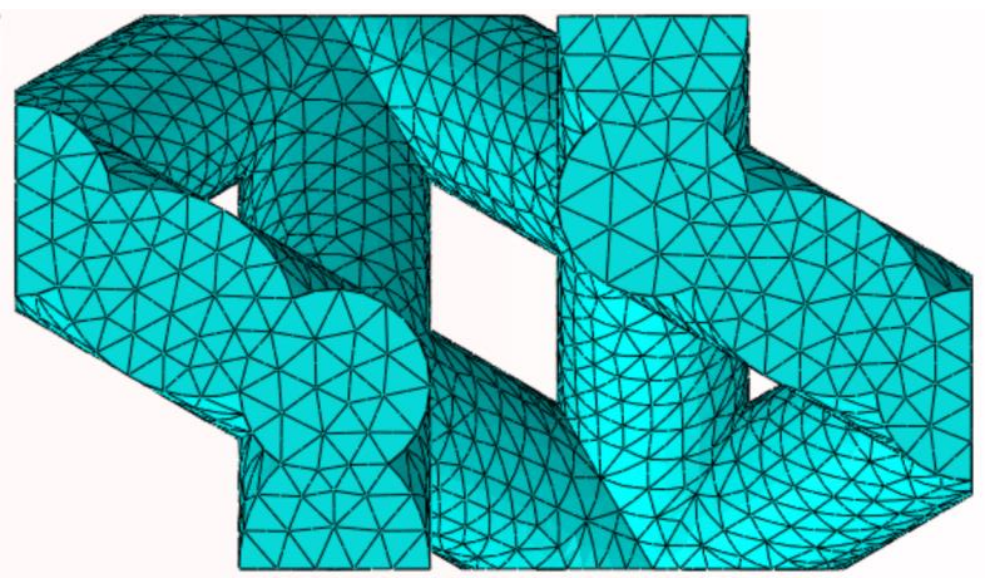

Figure 3: A sample of meshed REV, using C3D10 element type (10-node quadratic tetrahedron).
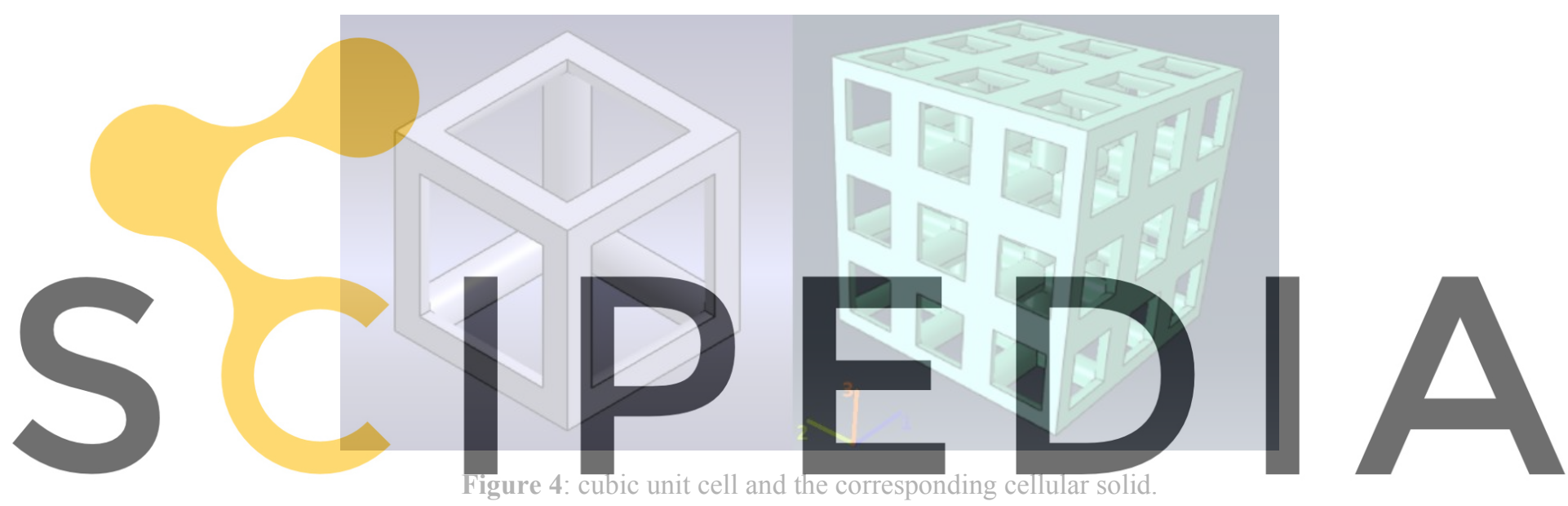

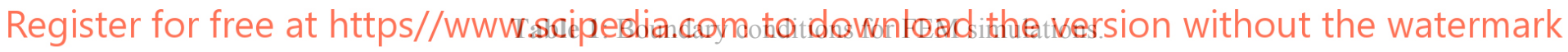

\begin{tabular}{|c|c|c|}
\hline $\begin{array}{l}\text { Output } \\
\text { Considered }\end{array}$ & $\begin{array}{l}\text { Applied boundary } \\
\text { conditions }^{*}\end{array}$ & Explanatory illustration \\
\hline \multirow{5}{*}{$\begin{array}{l}\mathrm{E}_{\mathrm{i}} \text { : principal } \\
\text { Young's modulus } \\
\text { in } \overline{\boldsymbol{i}} \text { direction }\end{array}$} & $\begin{array}{c}\text { Face }-\overline{\boldsymbol{i}} \rightarrow \text { Load } \\
\text { (normal to } \overline{\boldsymbol{j} \boldsymbol{k}} \text { plane) }\end{array}$ & \multirow{5}{*}{ symment } \\
\hline & $\begin{array}{c}\text { Face }+\overline{\boldsymbol{i}} \rightarrow \text { Symmetry } \\
(\text { plane: } \overline{\boldsymbol{j} \boldsymbol{k}})\end{array}$ & \\
\hline & $\begin{array}{c}\text { Face }+\overline{\boldsymbol{j}} \rightarrow \text { Symmetry } \\
\text { (plane: } \overline{\boldsymbol{k} \boldsymbol{i}})\end{array}$ & \\
\hline & $\begin{array}{c}\text { Face }+\overline{\boldsymbol{k}} \rightarrow \text { Symmetry } \\
(\text { plane }: \overline{\boldsymbol{i}})\end{array}$ & \\
\hline & Faces $-\overline{\boldsymbol{j}} \&-\overline{\boldsymbol{k}} \rightarrow$ Free & \\
\hline
\end{tabular}

*Note: the orientation of $\boldsymbol{i} \overline{\boldsymbol{j} \boldsymbol{k}}$ local coordination is located at the center of the unit cell. 


\section{RESULTS}

To investigate the effect of the architecture of the presented cellular solid the dimensionless ratio $b / a$ is defined which is related to the helical angle, $\phi=\tan ^{-1}(b / a)$. Increasing this parameter tilts the helical struts along direction 3. The Percentage of porosity, $p$, is defined as the ratio of the volume of pores to the volume of surrounding hexagonal prism and it is controlled by changing the diameters of struts, $d$. Dimensionless stiffnesses $S_{1}, S_{2}$, and $S_{3}$ are introduced as the ratio of the stiffnesses of cellular solid to the stiffness of the bulk counterpart $[2,5]$.
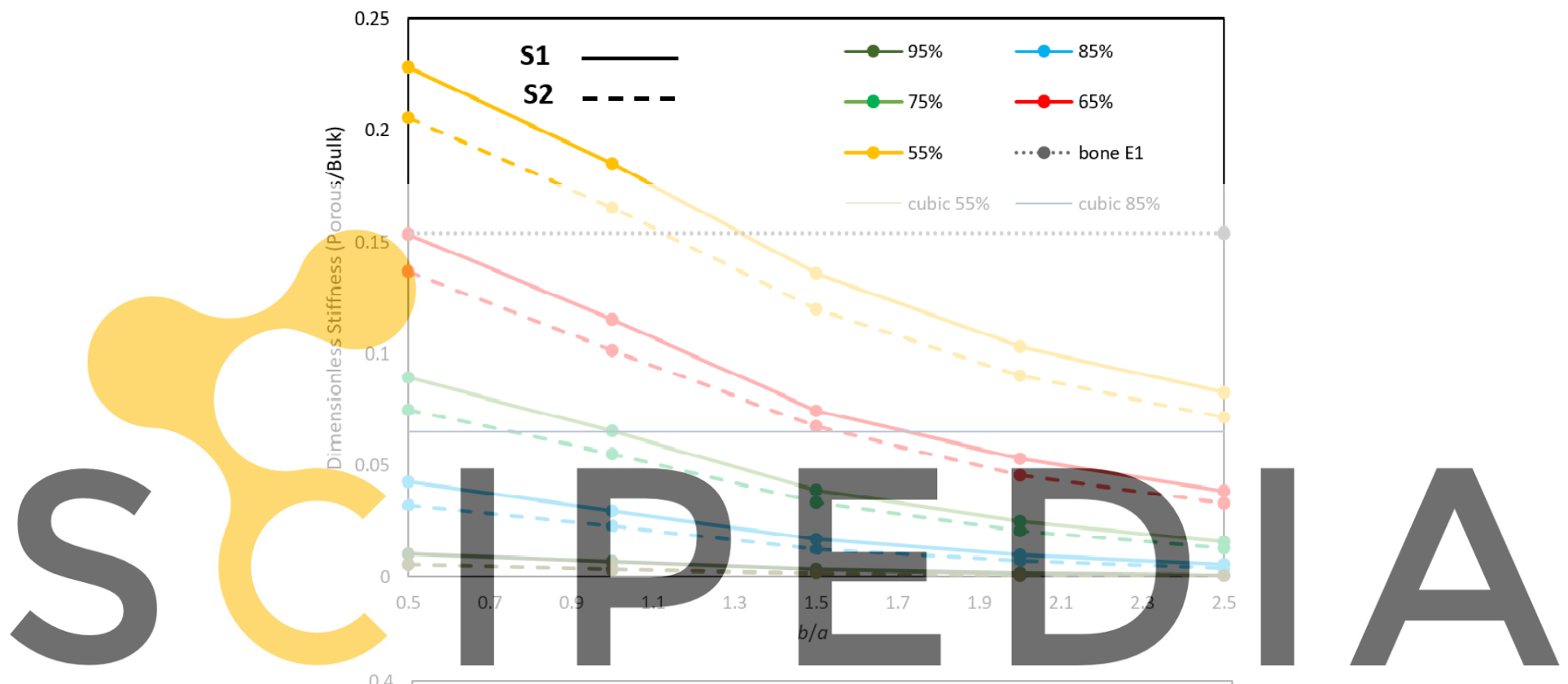

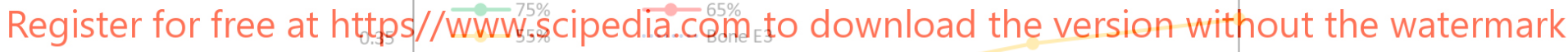

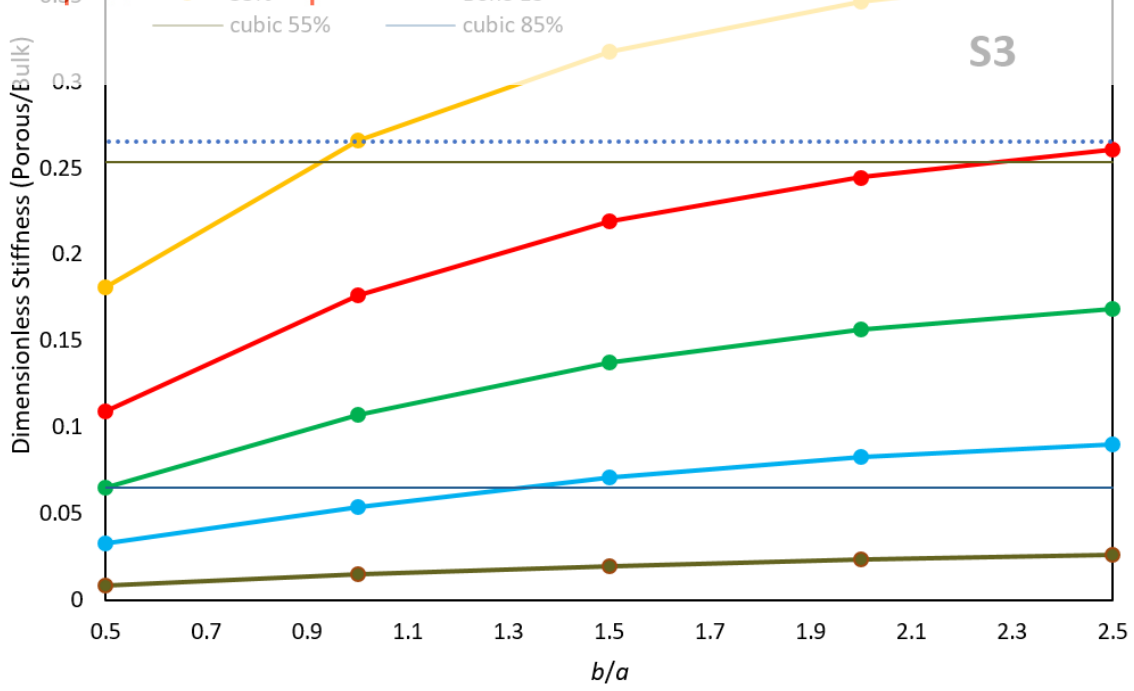

Figure 5: Dimensionless stiffness versus the dimensionless $b / a$ ratio. a) In-plane stiffness b) Out of plane stiffness. The dimensionless stiffness of bone from Reisinger et al. results [4] and cubic cellular solids for two percentages of porosity (55\% and $85 \%)$ are also depicted. 
Fig. 5 presents the dimensionless stiffnesses versus the dimensionless $b / a$ ratio for different values of the percentage of porosity, $p$. As expected, by increasing the porosity percentage, all three dimensionless stiffnesses reduce. One can observe that the in the in-plane stiffnesses, $S_{1}$, and $S_{2}$ are almost similar and reduce by increasing the dimensionless ratio, $b / a$ while the outof-plane stiffness, $S_{3}$, increases. The reason is that increasing $b / a$ tilts the helical struts along the out-of-plane direction 3. It means the helical angle, $\phi$, can be used as a tuning parameter to control the anisotropy of the presented cellular solid. Comparing to cubic cellular solids, the inplane stiffnesses of Isotruss cellular solid are lower than cubic ones, while, for the out-of-plane, it is possible to achieve higher values by selecting a proper helical angle. It is also seen the introduced cellular solid made by Ti6A14V alloy as the bulk material can present the stiffnesses in the range of bone elastic properties.

\section{REFERENCES}

[1] L. R. F. David W. Jensen, "Iso-truss structure". Patent EP1358392B1.

[2] Lorna J. Gibson, and Michael F. Ashby, Cellular Solids: Structure and Properties, 2nd ed., Cambridge: Cambridge University Press, 1997.

[3] Mathieu Dumas, Patrick Terriault, And Vladimir Brailovski, "Modelling and characterization of a porosity graded lattice structure for additively manufactured biomaterials," Materials \& Design, vol.121, pp. 383-392, 2017.
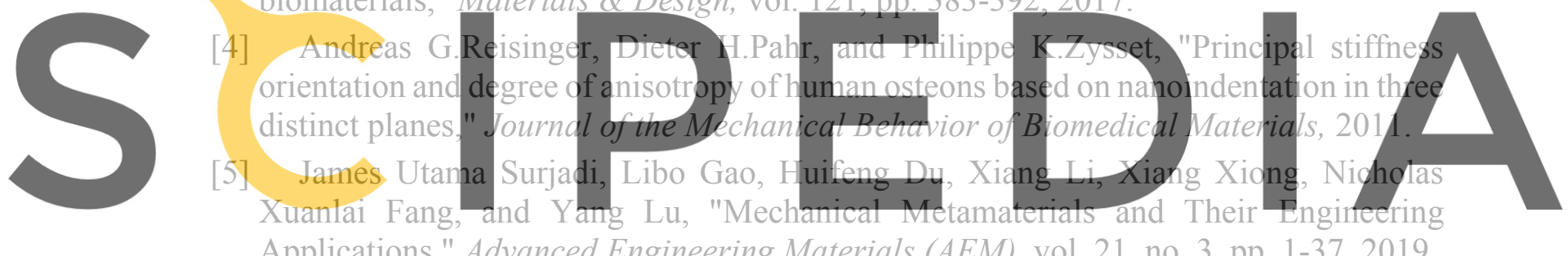

Applications," Advanced Engineering Materials (AEM), vol. 21, no. 3, pp. 1-37, 2019. 